\title{
Writing a paper for publication
}

\author{
Lesley Rees ${ }^{1} \cdot$ Michel Baum ${ }^{2}$ \\ Received: 31 July 2018 / Accepted: 2 August 2018 / Published online: 14 August 2018 \\ (C) IPNA 2018
}

\section{Introduction}

As editors of Pediatric Nephrology, we are often asked to provide assistance to potential authors on how to write a manuscript. We submit this brief editorial to provide some guidelines that authors may find helpful. Around 800 papers per year are submitted to Pediatric Nephrology and from these we select about one third for publication. What is it that motivates us as doctors to undertake and publish research? The prime motivation is to investigate new ideas that will improve scientific understanding and the care of patients. By publishing the data we disseminate the results; any discovery/ development that is not published is wasted and has let our patients down. Publications are also one way that academics communicate. If the papers are good, they also demonstrate the standing of the author in the academic community.

\section{Preparing the study}

When starting a study certain important principles need to be followed. Always ensure a clear hypothesis. This leads to clarity of your thought processes thereafter. Follow the principles of 'Good Clinical Practice', i.e. respect for the patient and their family, including ethical considerations and consent. Good Clinical Practice is an international standard for clinical research. Compliance with Good Clinical Practice provides public assurance that the rights, safety and wellbeing of research participants are protected and that research data are

Lesley Rees

Lesley.rees@gosh.nhs.uk

Michel Baum

Michel.Baum@UTSouthwestern.edu

1 Renal Office, Gt Ormond St Hospital for Children NHS Foundation Trust, London WC1N 3JH, UK

2 Department of Pediatrics, University of Texas Southwestern Medical Center at Dallas, Dallas, TX, USA reliable. Make sure that you have Institutional Review Board (IRB) approval before embarking on a study. The use of public/charity funding and involvement of families obliges us to ensure that our study is realistic. Can the following criteria be fulfilled?

Is the question:

- meaningful

- specific

- answerable in general

- answerable in this study

A power calculation is essential before you start your study, as without one it may be meaningless and without any scientific value.

Many researchers follow the PICO process when developing a new evidence-based study or systematic review. PICO is a mnemonic for the important parts of the study:

PICO

Patient, Problem or Population. e.g. age, gender, comorbidity

Intervention. e.g. tests, treatment, exposure

Comparison, Control or Comparator: e.g. none, placebo, gold standard

Outcome

\section{Writing the paper}

\section{Some general rules}

State your hypothesis clearly so that it is obvious to the reader why you have done the study. We suggest that authors start by writing the results first, then the methods, the discussion and the conclusion followed by the introduction. Leave the abstract and title until the last. Follow the journal instructions 
to authors carefully and avoid abbreviations unless they are well known and well recognised ones.

\section{Results}

This is the crux of the paper and the most important section from which the rest will follow so start with this section first. Use tables and figures as much as possible; there is no need to duplicate tables/figures in the text. Ensure that figure legends cover all aspects and fully enable understanding of their purpose. The number of patients, samples or assays should be provided as well as whether differences are statistically significant. Ensure that all images are of high quality and are worthy of your findings. The results section is not where you discuss interpretation of results; that is for the discussion.

\section{Methods}

The methods should be in enough detail for the reader to be able to repeat the study themselves. There should be an accompanying statement of ethical approval and patient/parental consent. The statistics used to analyse your results must be detailed and a power calculation included.

\section{Discussion}

The first few sentences should summarise your findings. Here is the place to discuss the significance of your results but do not repeat them. Discuss how your data fit in with the published literature. If a confirmatory study, what makes yours different and original? Every statement should be referenced.

It is important to have a paragraph to discuss the limitations of the study. Take care not to repeat this in the introduction. Do not make claims that are not justified by your results or are not part of the study.

\section{Conclusion}

Finish the discussion with a conclusion. This can be as short as two or three sentences. Do not make claims that are not justified by your results or are not part of the study. Many papers finish with 'further research is needed'. We usually know this and it is not helpful.

\section{Introduction}

State your hypothesis clearly. Say where your work fits into the field as whole. Say why the study is needed, particularly if it is comparable to previous work. Keep it brief. Ensure that there is no duplication between the introduction and the discussion.

\section{Abstract}

Each journal has an abstract structure and word limit. You are usually asked to break the abstract down into background, methods, results and conclusion. Care is needed for a good abstract: if the reader is not interested after reading the abstract, they will not read the paper!

\section{Title}

By the time the paper is written, the choice of title will become much clearer. It must be interesting, brief and accurate; there is usually a word restriction.

\section{Authors}

You will need to decide who has contributed to the work and decide in what order to list them. It is vital that they have all played a significant role, have read the paper and agreed with its contents.

\section{References}

Ensure every fact you discuss is referenced using the most up to date reference. Use journals rather than books, as books can be difficult for readers to obtain. In addition, the information in books comes from original articles. Use the correct format: if incorrect, this suggests to the editor that the paper has been submitted elsewhere already and has been rejected. Check carefully that the right reference has been used for each fact. Programmes such as End note/Reference manager are helpful.

\section{The 'dos' and 'don'ts'}

\section{The don'ts}

Do not make careless mistakes such as poor spelling and grammar, misnumbering of figures and tables and/or references in the wrong place or not in journal style. This suggests to the editor and reviewers that the work is 'sloppy' and that there may be errors in the methods and results as well.

Do not over interpret conclusions; this will shake the scientific foundation of the paper.

Do not make statements without references or miss important references. It is likely that an author on one of these important papers will be reviewing your paper!

Editors check for plagiarism using iThenticate. This compares your text with texts from $>126$ publishers and $>50,000$ journals. It will even pick up plagiarism of your own work.

Finally, abbreviations make a text very difficult to follow and sometimes unreadable. Some abbreviations are established: e.g. CKD, AKI and these are acceptable. 


\section{The dos}

Do read the instructions to authors and follow them; do not make the editor think they are second (or more!) choice. Be brief but complete. The most important is a first class presentation and analysis of the results. Do not be ashamed to ask for help: discuss with your senior colleagues and follow their advice. If English is not your first language, enlist the assistance of a colleague proficient in English to read and revise your manuscript.

\section{Where should I submit my paper?}

You will need to decide if the paper should be sent to a speciality or a subspecialty journal. Take advice from senior colleagues on this. Do you want to maximise the audience for your paper? e.g. a paediatric nephrology paper is most likely to be read by paediatric nephrologists in the journal Pediatric Nephrology. However, many academics with a strong piece of research will want to publish in a journal with the highest possible impact factor. This is because they will be judged academically by the number of papers they have in high impact factor journals.

\section{The process for Pediatric Nephrology}

\section{What happens after the paper is submitted?}

One of the two editors or an associate editor (for an educational article or review) will read the paper and decide whether to immediately reject it or send it out to reviewers. About half of the submitted manuscripts are not sent out for review at this stage. Reviewers are selected who are expert in the area. They advise the editor on the quality of the paper and the priority for publication. They provide you with helpful advice on the ways to improve your paper.

\section{What are the editors looking for?}

That there is a statement that ethical permission and informed consent have been obtained, and that there is a statement of 'Conflict of interest'.
Is there a clear hypothesis? Was the study well conducted? If this is a repeat of previous work, why is it needed? Are the statistics sound and is there a power calculation? Is the paper meaningful to the international paediatric community, i.e. it is not just a local audit. Is it readable?

A paper is likely to be excellent if it is novel, timely, meaningful and will influence thinking or practice.

\section{Letter of response to reviewers}

When all the reviews are received the editor decides whether to accept the paper straight away, to reject straight away or whether to send back for revision. The author should realise that the reviewers also write a confidential letter to the editor that affects the decision. If revision is needed a letter is sent to the authors with all the reviewers' comments.

The authors must then revise their paper, answering all the questions asked by the reviewers. It is important to treat each point carefully and with respect. To help the reviewers when they receive your resubmission, answer each comment in turn and rewrite the comment followed by your response in full. Identify the response in the commentary and in the text, even if this appears to be repetitive. You are entitled to rebut a criticism if you have a good argument against it. Your response to the reviewers and the new paper with identified changes within will then go back to the reviewers for further comment. More than one revision may be necessary. Ultimately the editor will decide whether the authors have adequately addressed the reviewers' comments and accept or reject the paper.

\section{What if my manuscript is rejected?}

Do not be despondent: all the reviewers may not all hold the same opinion (they are the jury) and the editor (acting as the judge) has to make a final decision. The paper can be reassessed by other journals with the benefit of positive changes made by the authors following the reviews. Persistence is part of academic medicine. We are not looking to reject your papers. We would like to encourage you to send them! 